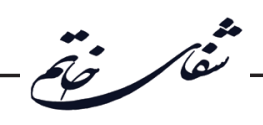

\title{
Explanation of Psychological Problems Based on Cognitive Flexibility and Self Differentiation
}

\author{
Hossein Eskandari ${ }^{1}$, Shima Pajouhinia ${ }^{{ }^{*}}$, Yalda Abavisani ${ }^{2}$ \\ ${ }^{1}$ Psychological Department, Education and Psychology Faculty, Allameh Tabataba'i University, Tehran, Iran \\ ${ }^{2}$ Counseling Department, Education and Psychology Faculty, Quchan Branch, Islamic Azad University, Quchan, Iran
}

\section{A BSTRACT}

Introduction: cognitive flexibility and self-differentiation are the appropriate models for understanding psychological problems through which people can meet various challenges. The aim of this study was to investigate the relationship between cognitive flexibility and self-differentiation with psychological problems among female students in Allameh Tabataba'i University. Materials and Methods: The method of this study was descriptive-correlational and statistical population was comprised of all female students at AllamehTabataba'i University in Tehran who have been studying between 2014-2015. Using random multiple cluster sampling method, a group of 380 students were selected. Tools were questionnaires of cognitive flexibility, self-differentiation and symptoms checklist (SCL90-R). Data were analyzed by descriptive statistical parameters, correlation coefficient, and regression analysis. Results: Analysis revealed a significant correlation between total score of psychological problems with cognitive flexibility and self-differentiation. In addition, the results of multiple regression analysis showed that cognitive flexibility and self-differentiation can explain different kinds and levels of psychological problems in students. Conclusion: study of cognitive flexibility and self-differentiation are efficient methods to understand the underlying factors associated with psychological problems. Evaluation of these factors can

\section{Key words:}

1. Mental Health

2. Resilience,Psychological 3. Cognition lead to a new approach in prevention and treatment of psychological problems.

*Corresponding Author: Shima Pajouhinia

E-mail: sh.pajouhinia@yahoo.com 
تبيين مشكلات روانى مبتنى بر انعطاف يذيرى شناختى و تمايزيافتكى خود

\author{
حسين اسكندرى'، شيما يُوهىنيا"، يلدا ابويسانى' \\ 'كروه روانشناسى، دانشكده علوم تربيتى و روانشناسى، دانشكاه علامه طباطبائى، تهران، ايران

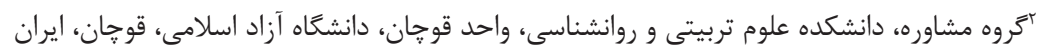

كليد وازهها:

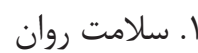

r. انعطاف گيذيرى روانى

r.

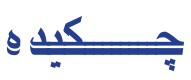

مقدمه: انعطاف يذيرى شناختى و تمايزيافتگى خود مدلهاى مناسبى براى شناخت مشكلات روانى

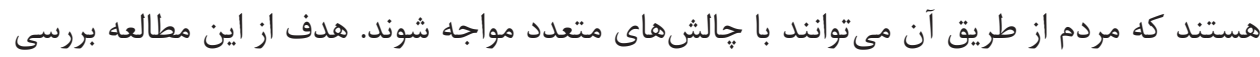

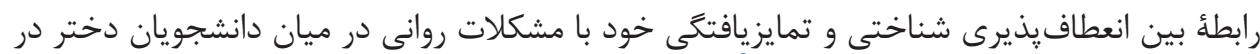

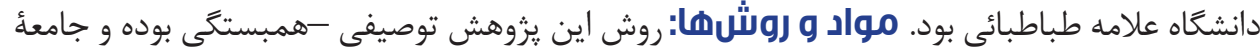

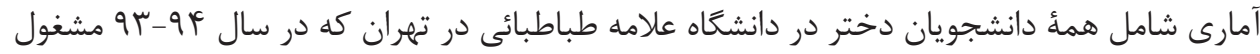

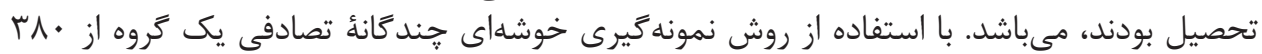

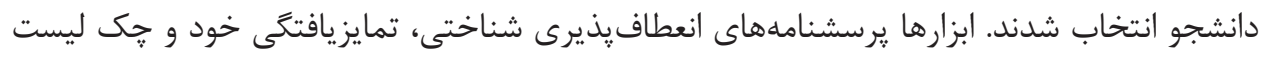

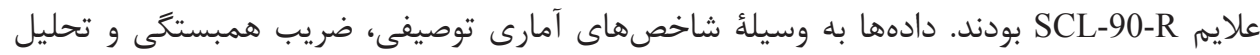

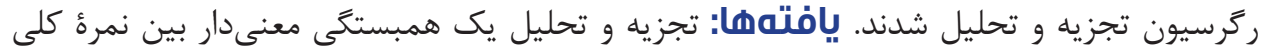

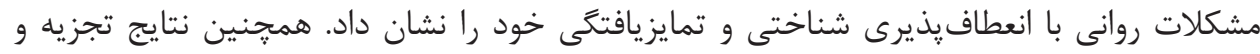

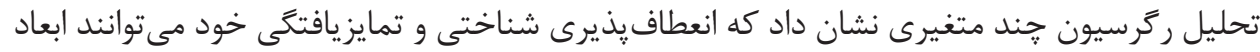

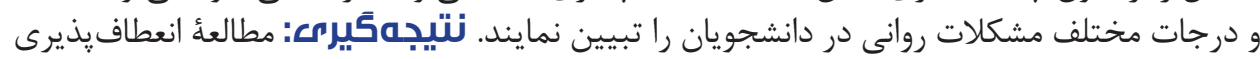

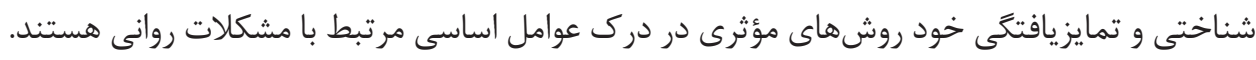

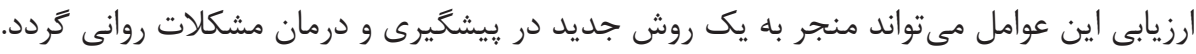




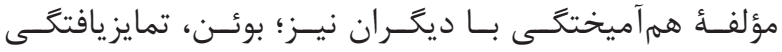

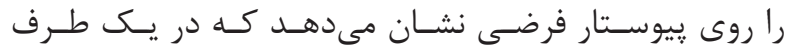

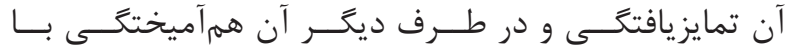

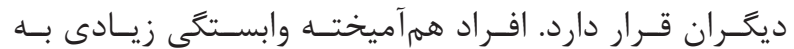

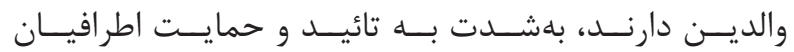

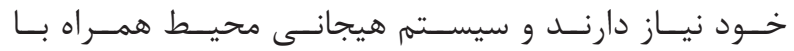

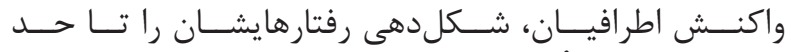

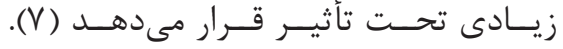

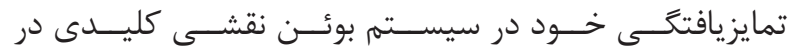

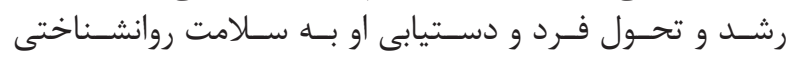

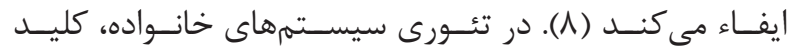

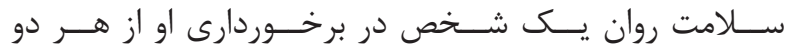

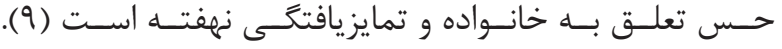

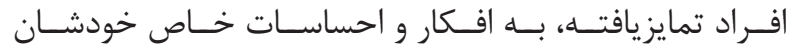

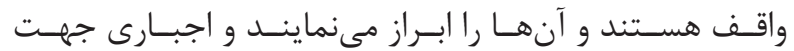

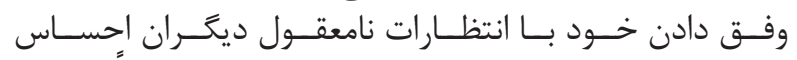

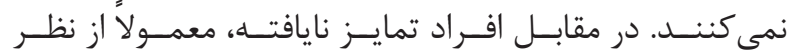

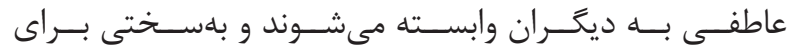

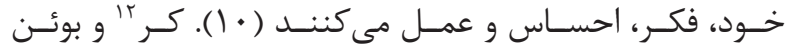

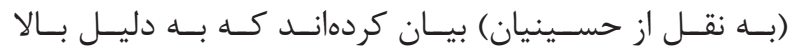

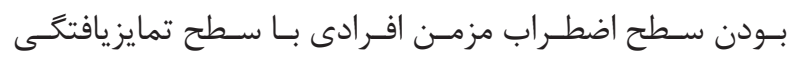

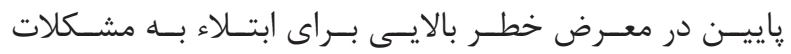

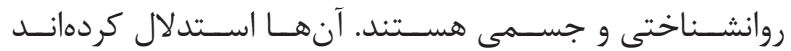

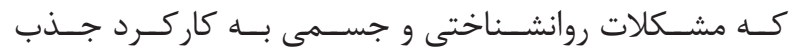

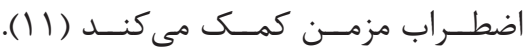

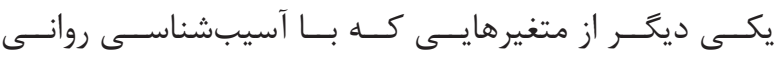

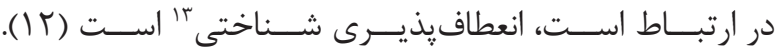

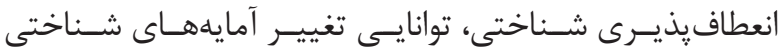

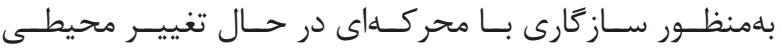

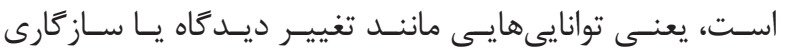

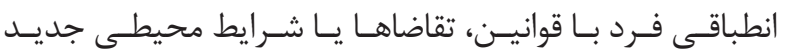

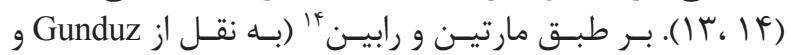
فeBerry

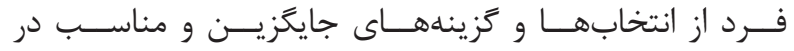

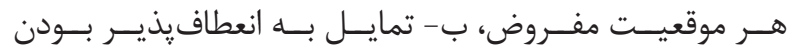

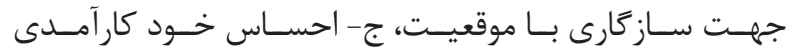

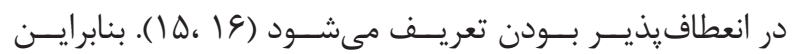

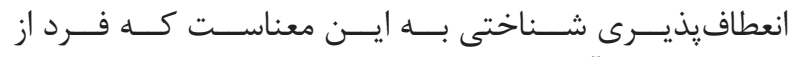

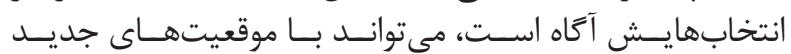

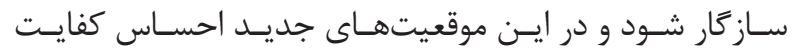

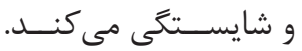

مطابـق بــا نظريـــُ انعطاف قِذيــرى شـــاختى، ســلامت روان

${ }^{1}$ Vulnerability

${ }^{2}$ Invulnerability

${ }^{3}$ Diathesis- stress model

${ }^{4}$ Differentiation of self

${ }^{5}$ Bowen

${ }^{6}$ Interapsychi

${ }^{7}$ Interpersonal

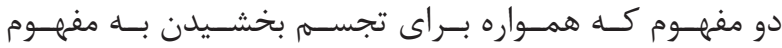

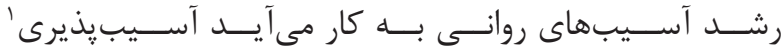

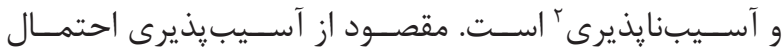

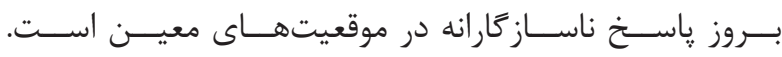

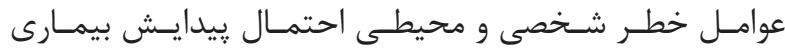

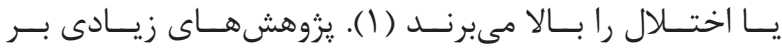

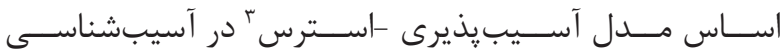

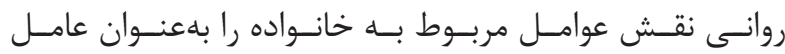

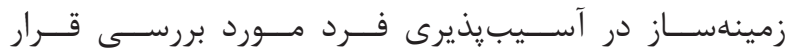

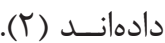

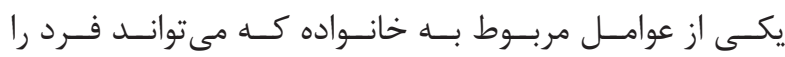

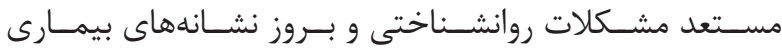

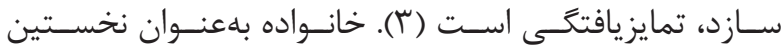

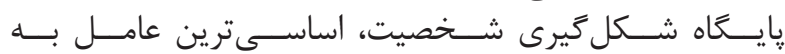

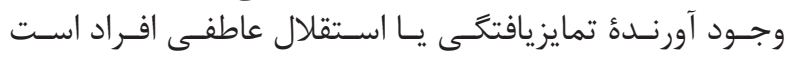

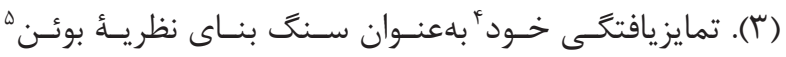

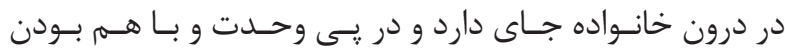

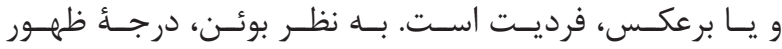

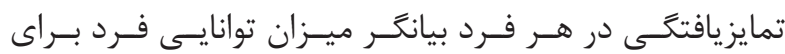

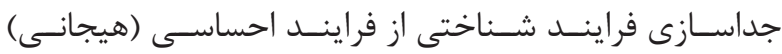

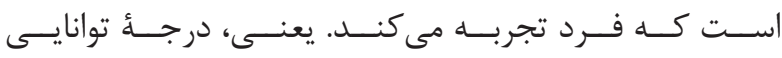

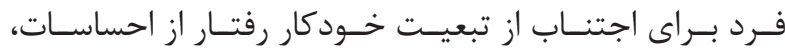

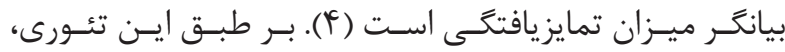

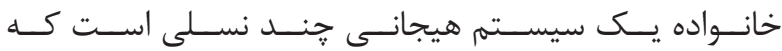

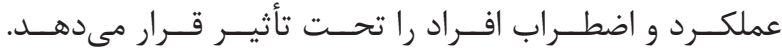

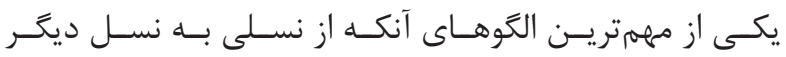

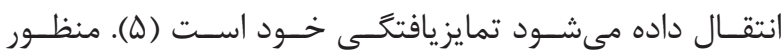

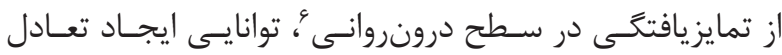

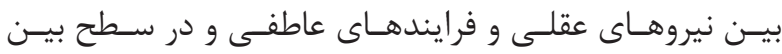

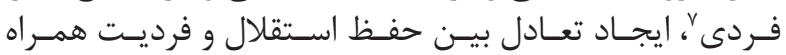

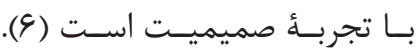

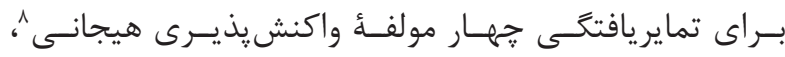

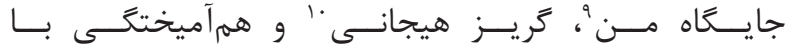

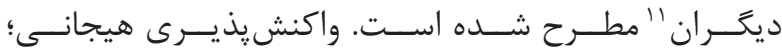

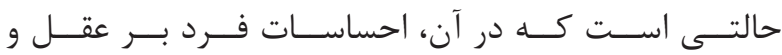

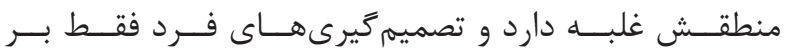

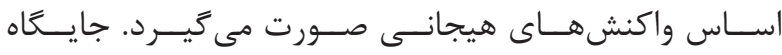

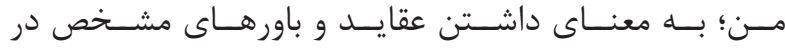

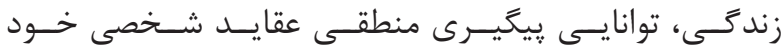

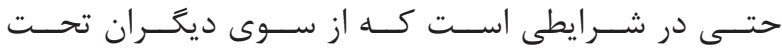

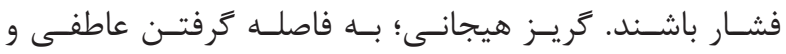

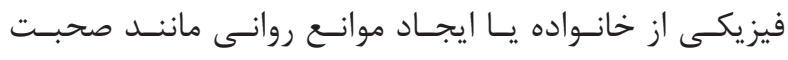

${ }^{8}$ Emotional reactivity

${ }^{9}$ I- position

${ }^{10}$ Emotional cut off ${ }^{11}$ Fusion with others

${ }^{12}$ Kerr

${ }^{13}$ Cognitive flexibility

${ }^{14}$ Martin and Rubin 


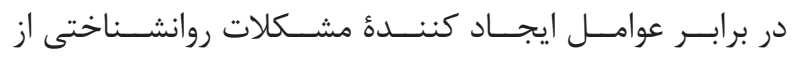

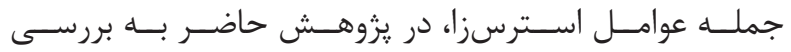

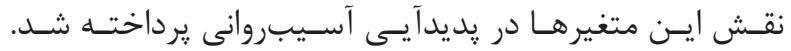

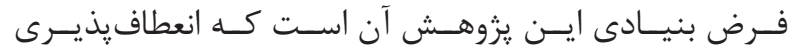

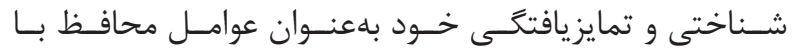

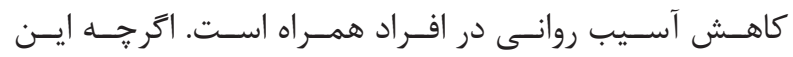

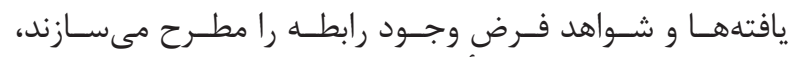

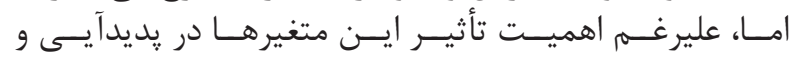

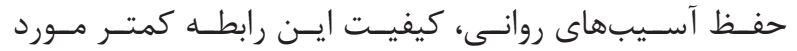

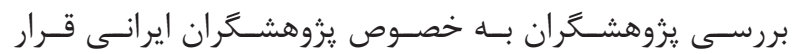

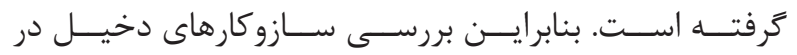

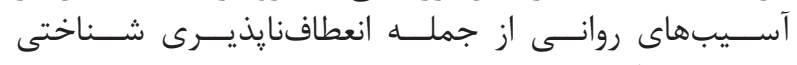

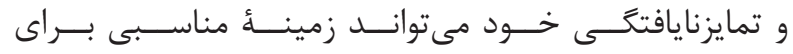

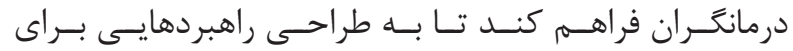

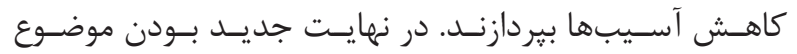

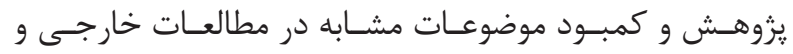

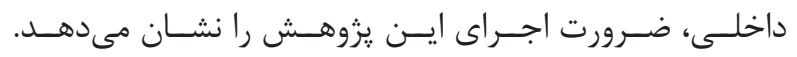

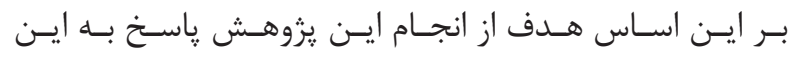

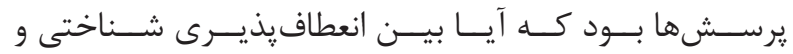

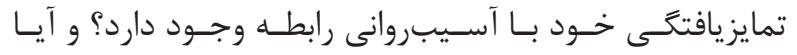

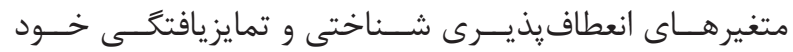

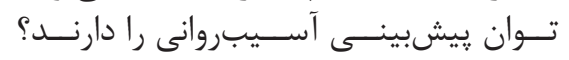

مواد و روشهان

روش :زثوهش، جامعلُ آمارى و نمونه

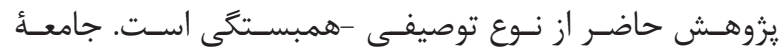

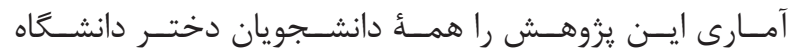

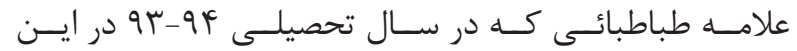

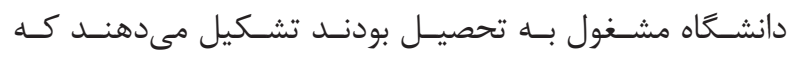

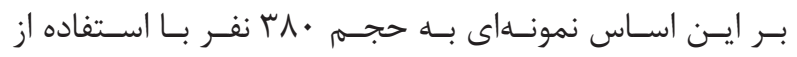

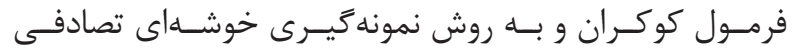

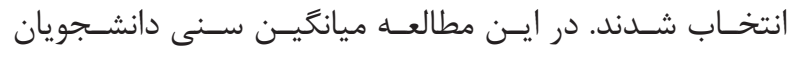

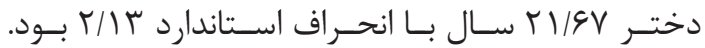

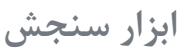

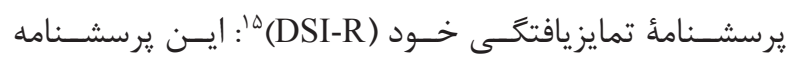

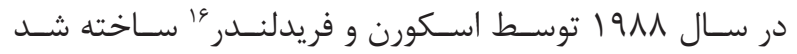

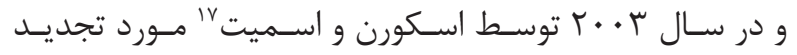

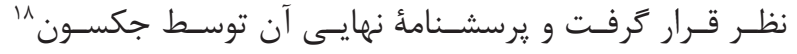

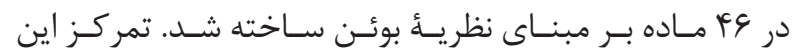

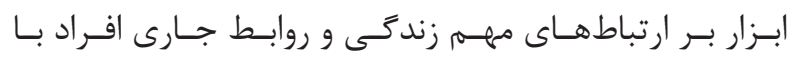

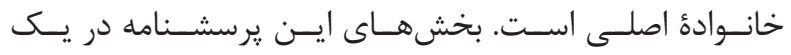

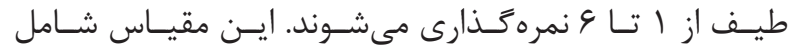

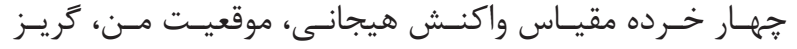

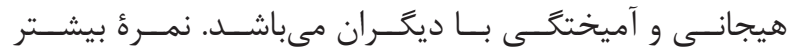

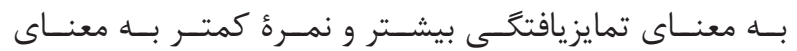

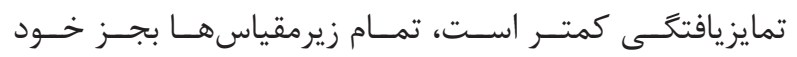

${ }^{15}$ Differentiation of self inventory-revised

${ }^{16}$ Skowron and Friedlander

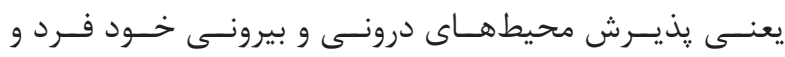

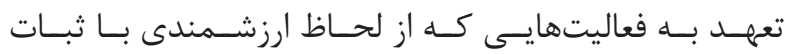

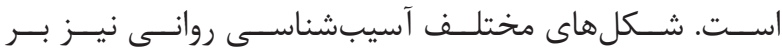

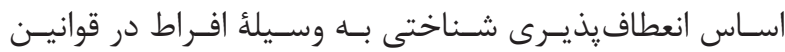

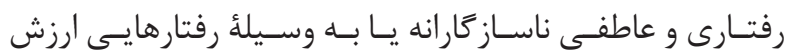

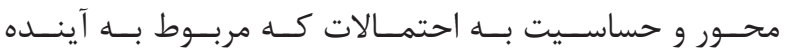

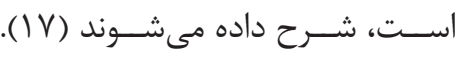

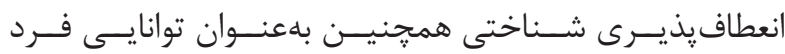

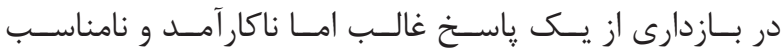

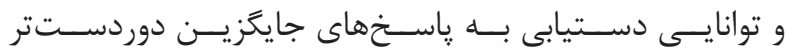

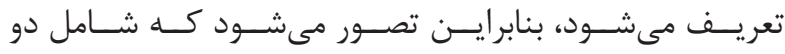

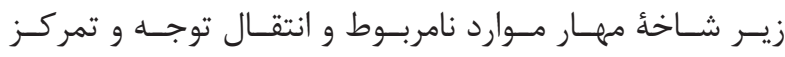

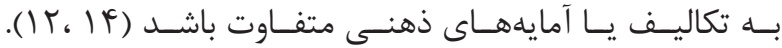

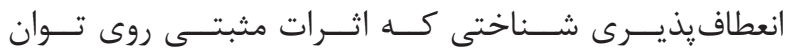

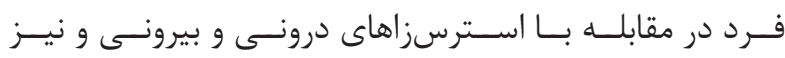

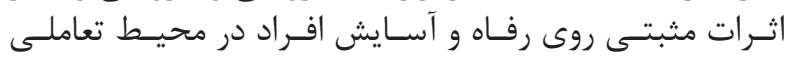

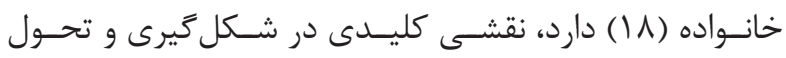

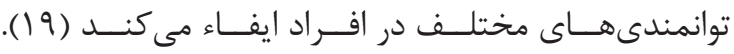

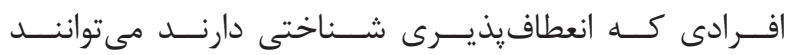

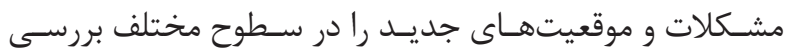

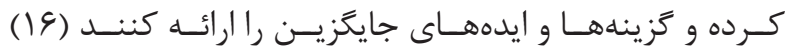

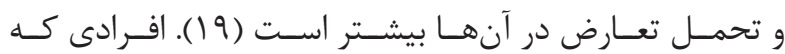

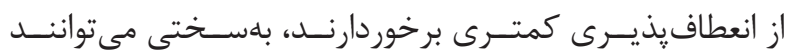

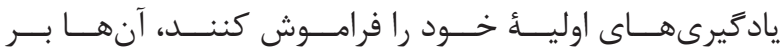

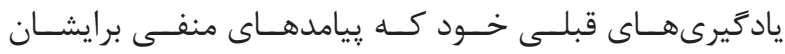

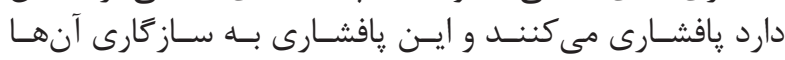

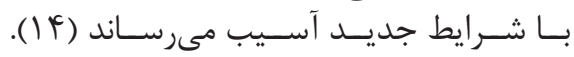

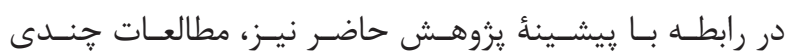

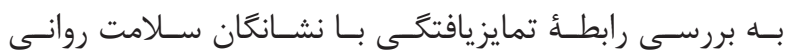

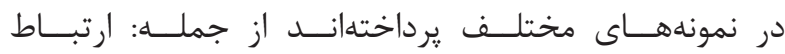

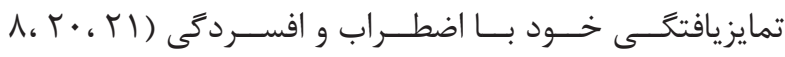

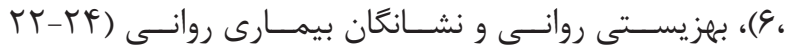

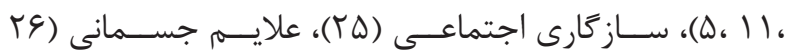

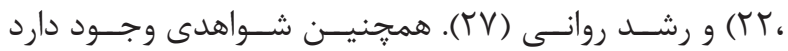

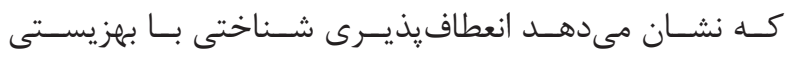

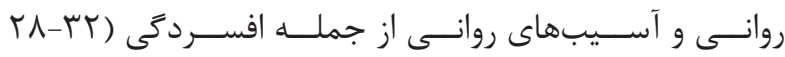

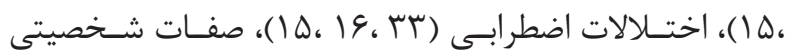

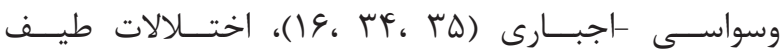

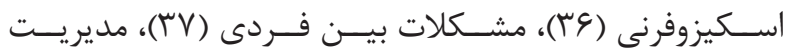

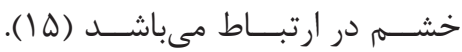

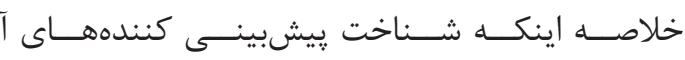

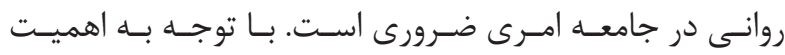

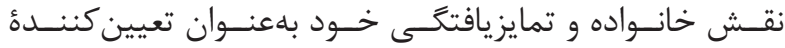

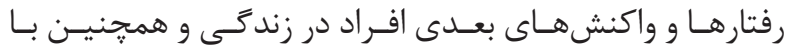

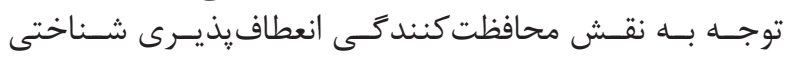

${ }^{17}$ Smith
${ }^{18}$ Jackson 


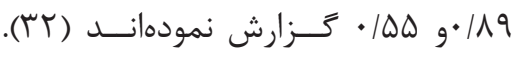

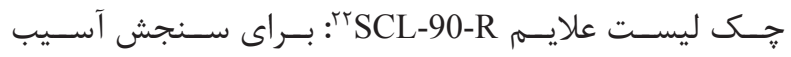

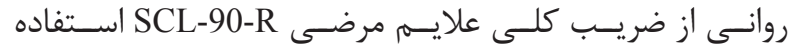

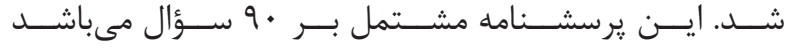

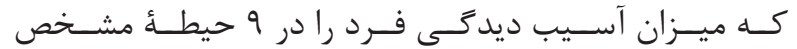

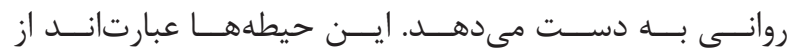

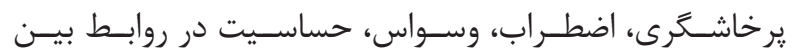

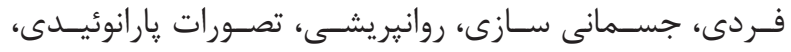

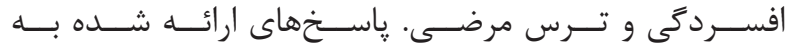

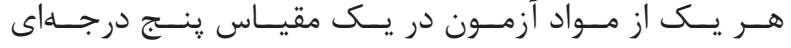

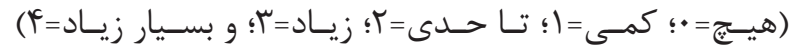

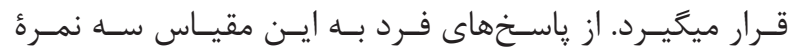

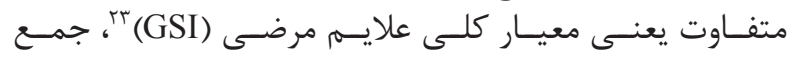

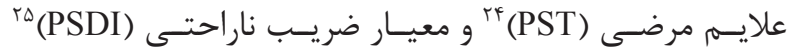

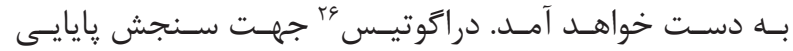

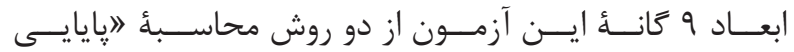

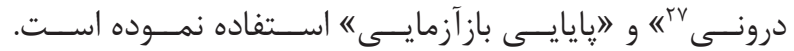

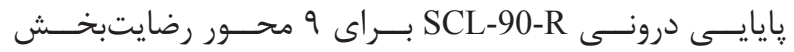

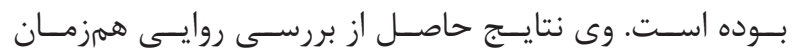

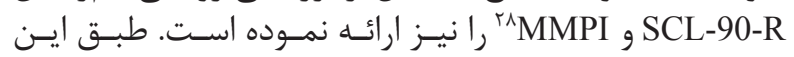

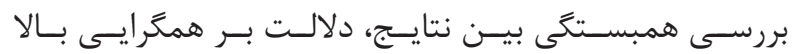

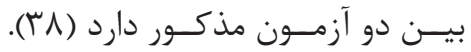
تجزيه و تحليل دادهها

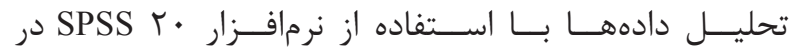

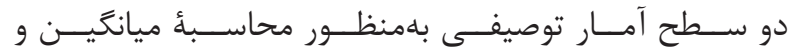

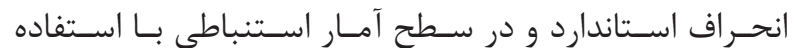

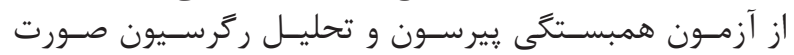

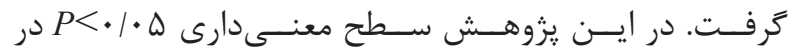

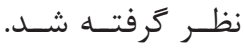

يافتهها

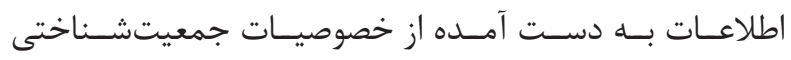

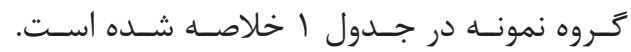

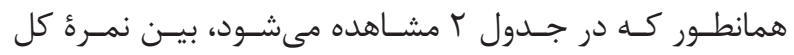

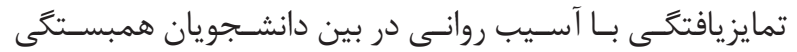

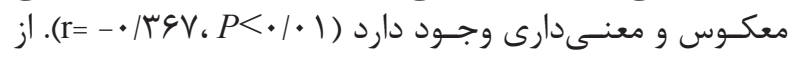

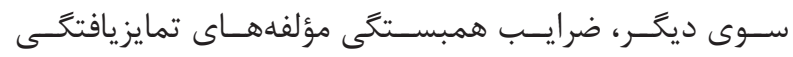

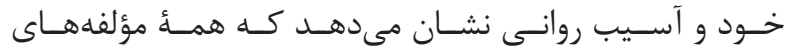

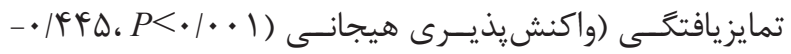

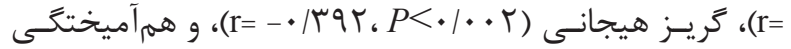

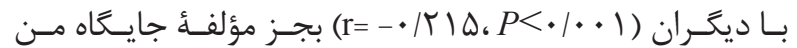

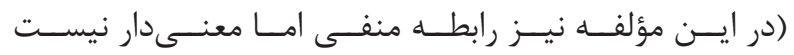

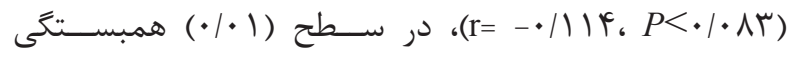

${ }^{19}$ Cognitive flexibility questionnaire

${ }^{20}$ Dennis and Vander wal

${ }^{21}$ Beck depression inventory-II

${ }_{22}^{2}$ Symptom checklist-90-revised

${ }^{23}$ Global symptom inventory

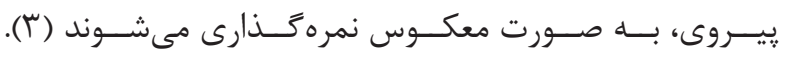

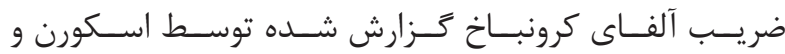

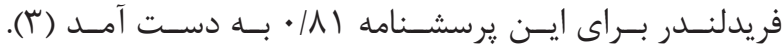

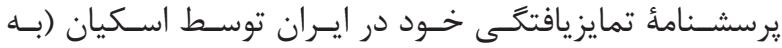

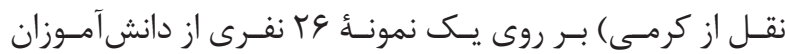

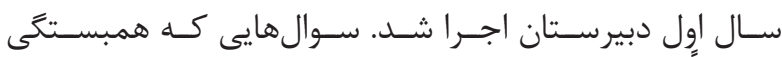

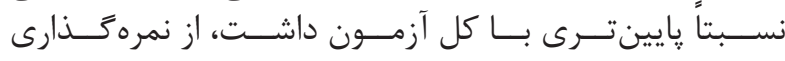

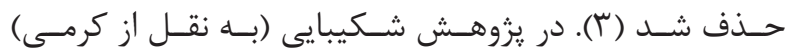

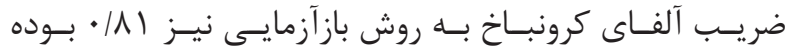

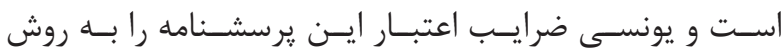

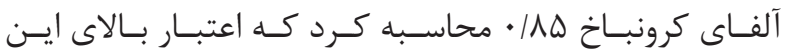

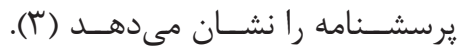

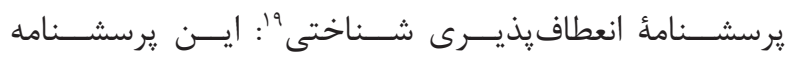

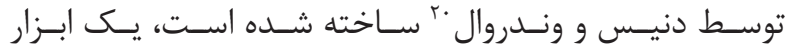

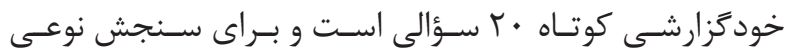

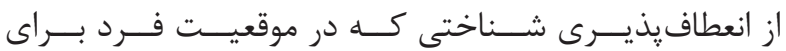

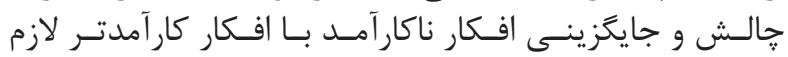

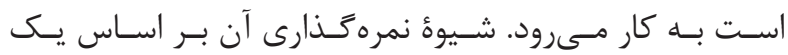

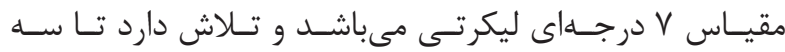

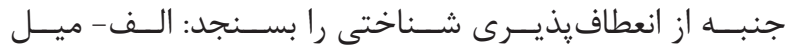

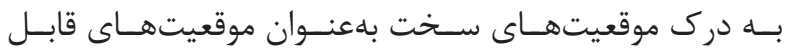

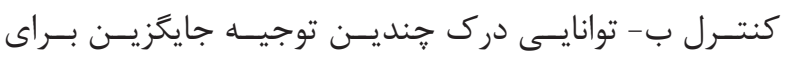

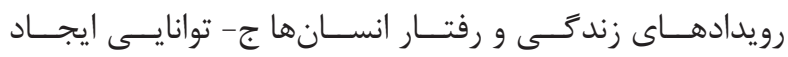

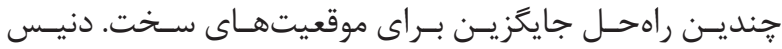

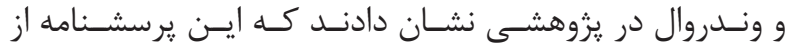

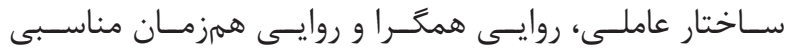

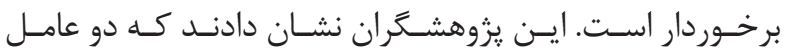

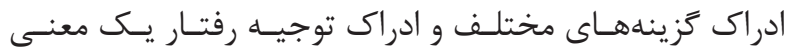

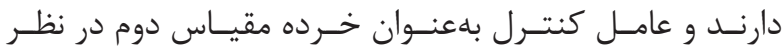

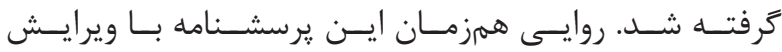

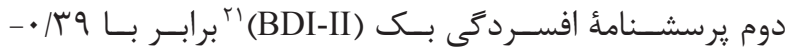

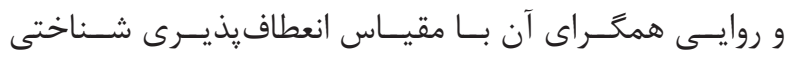

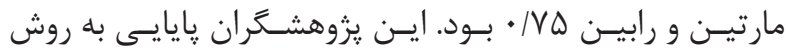

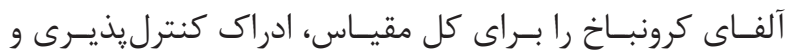

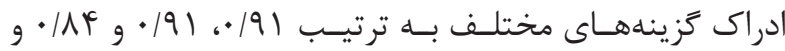

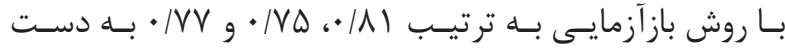

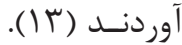

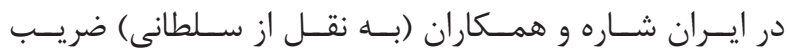

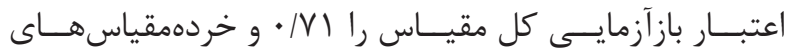

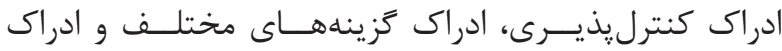

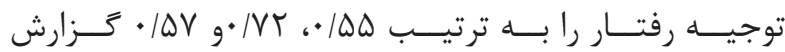

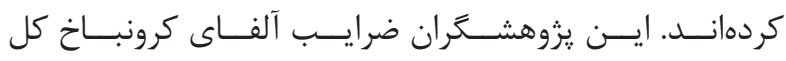

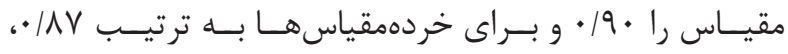

${ }^{24}$ Positive symptom total

${ }^{25}$ Positive symptom distress inventory

${ }^{26}$ Deragotis

${ }^{27}$ Internal reliability

${ }^{28}$ Minnesota multiphasic personality inventory 
جدول ا - اطلاعات جمعيتشناختى نمونه. - - إ.

اطلاعات جمعيتشناختى نمونه: تعداد (درصد)

\begin{tabular}{|c|c|c|}
\hline rᄉ $\cdot(/ .1 \cdots)$ & 「人. & فراوانى \\
\hline$r \wedge \cdot(/ .1 \cdots)$ & زن & جنس \\
\hline$Q V(/ / T \Delta / \Delta T)$ & كمتر از •r & \multirow{3}{*}{ سن } \\
\hline$r r \cdot(/ / \Delta V / \Lambda ৭)$ & بين •r تا ه广 & \\
\hline \&r $(/ .19 / 0 V)$ & بالاى ف r & \\
\hline$r \mid r(/ \Lambda r / 1 \cdot)$ & مجرد & \multirow{3}{*}{ تأهل } \\
\hline$f \mid(/ .1 \cdot / \vee q)$ & متأهل & \\
\hline$r V(/ . V / I \cdot)$ & مطلقه & \\
\hline
\end{tabular}

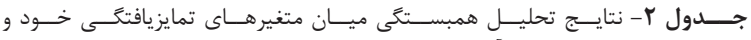

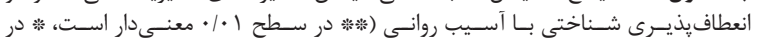

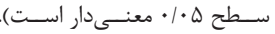

\begin{tabular}{|c|c|c|}
\hline آسيب رواتى & شاخص & متفيرهاى بيشبين \\
\hline$-\cdot / r q \Lambda * *$ & $\mathrm{R}$ & \multirow{2}{*}{ ادراى ثزينهماى مختلف } \\
\hline$\cdot 1 \cdots 1$ & $P$ & \\
\hline$-\cdot / r f q * *$ & $\mathrm{R}$ & \multirow{2}{*}{ ادراى كنترليذيرى } \\
\hline$\cdot 1 \cdots 1$ & $P$ & \\
\hline$-\cdot / 1 \mathrm{Vr} * *$ & $\mathrm{R}$ & \multirow{2}{*}{ ادراى توجيه رفتار } \\
\hline$\cdot 1 \cdots 1$ & $P$ & \\
\hline$-\cdot / r f f * *$ & $\mathrm{R}$ & \multirow{2}{*}{ مرة كل انعطاف يذيرى شناختى } \\
\hline$\cdot 1 \cdots 1$ & $P$ & \\
\hline$-\cdot / f f \Delta^{* *}$ & $\mathrm{R}$ & \multirow{2}{*}{ واكنشيذيرى هيجانى } \\
\hline$\cdot \cdots 1$ & $P$ & \\
\hline$-\cdot / 11 f$ & $\mathrm{R}$ & \multirow{2}{*}{ 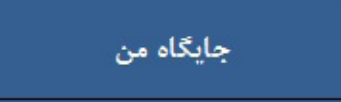 } \\
\hline $.1 \cdot 14$ & $P$ & \\
\hline$-\cdot / 494 * *$ & $\mathrm{R}$ & \multirow{2}{*}{ تمريز هيجانى } \\
\hline.$\cdots r$ & $P$ & \\
\hline$-\cdot / r 1 Q^{* * *}$ & $\mathrm{R}$ & \multirow{2}{*}{ هممآميختَّى با ديكَران } \\
\hline$\cdot 1 \cdots 1$ & $P$ & \\
\hline$-\cdot / 49 \mathrm{~V} * *$ & $\mathrm{R}$ & \multirow{2}{*}{ نمرة كل تمايزيافتكَى خود } \\
\hline 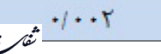 & $P$ & \\
\hline
\end{tabular}

جدول ب- نتايج تحليل ركرسيون كام به كام جهت بِيشبينى آسيب روانى از روى مؤلفههاى انعطافيذيرى شناختى (* آزمون در سطح ه • • معنى دار است).

\begin{tabular}{|c|c|c|c|c|c|}
\hline ضريب تعيين (R2) & سطح معنىىارى & آمارة & ضرايب استاندارد (Beta) & مدل ل & \\
\hline$\cdot / 1 \Delta \Lambda$ & $\cdot \cdots *$ & $-V / 11 Y$ & $-\cdot /$ \%१। & ادراى تزينههاى مختلف & مدل \\
\hline \multirow[t]{2}{*}{. /NA } & $.1 \cdots *$ & $-\Delta / \mu \cdot 1$ & $-\cdot / 4 / 8$ & ادراى كَزينههاى مختلف & \multirow{2}{*}{ 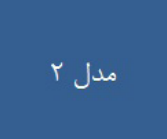 } \\
\hline & $\cdot \cdots *$ & $-r / 1 \cdot \Delta$ &.$- / 190$ & ادراى كنترليذيرى & \\
\hline. & $\cdot / \cdot 09$ & $-1 / \mu_{f}$ & $-\cdot / 1 \cdot \Delta$ & ادارى توجيه رفتارى & شتغيرهاى خارج \\
\hline
\end{tabular}

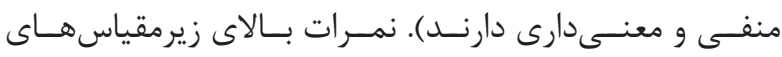

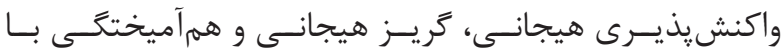

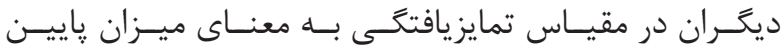

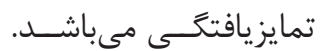

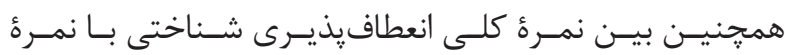

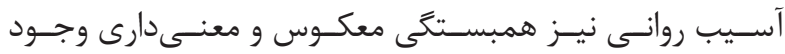

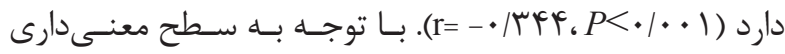

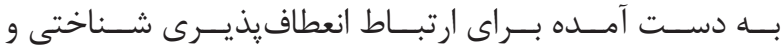

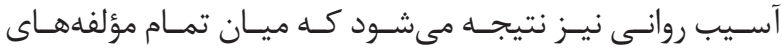

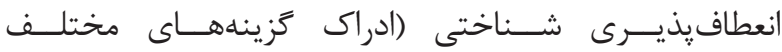

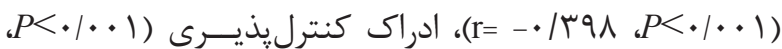

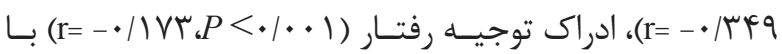

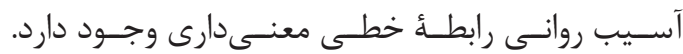

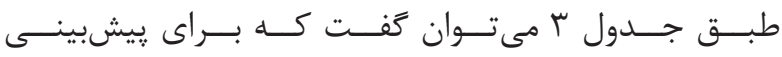

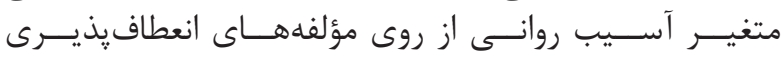

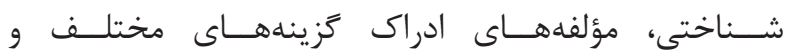

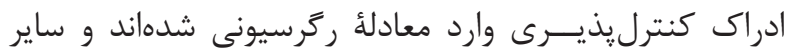

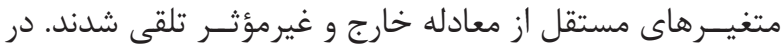

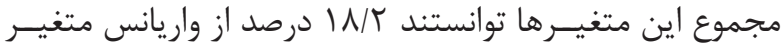

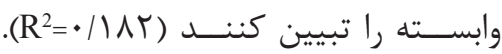

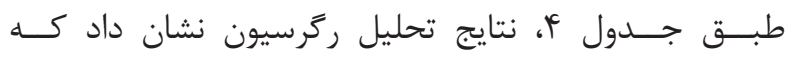

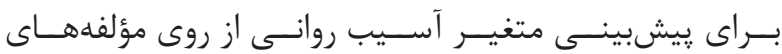

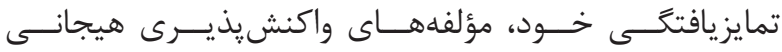

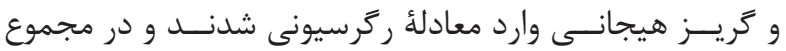

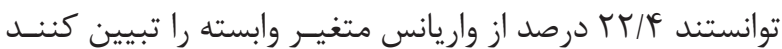

. $\left(\mathrm{R}^{2}=\cdot / T T Y\right)$

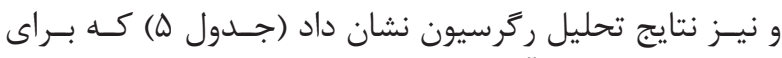

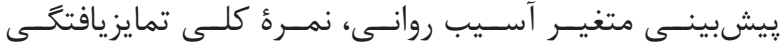

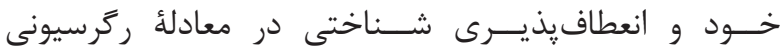

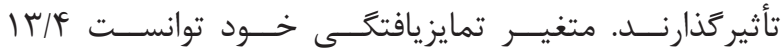

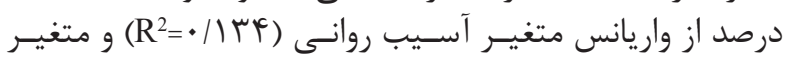

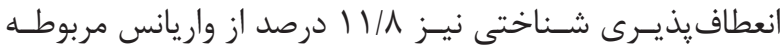

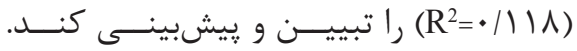




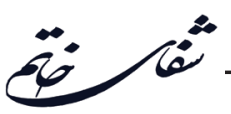

جدول F- نتايج تحليل ركرسيون كَام به كام جهت بِيشبينى آسيب روانى از روى مؤلفههاى تمايزيافتگى خود (** آزمون در سطح ه • • معنى دار است).

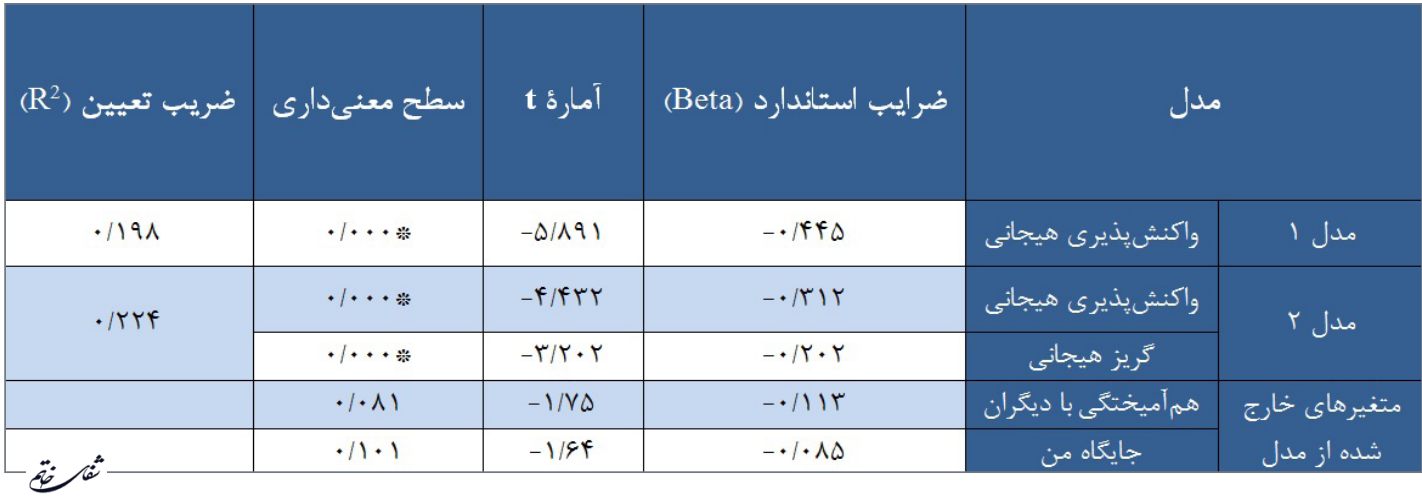

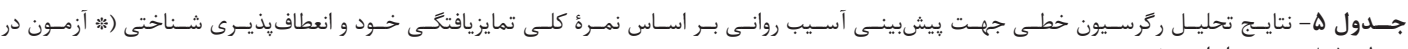

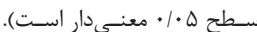

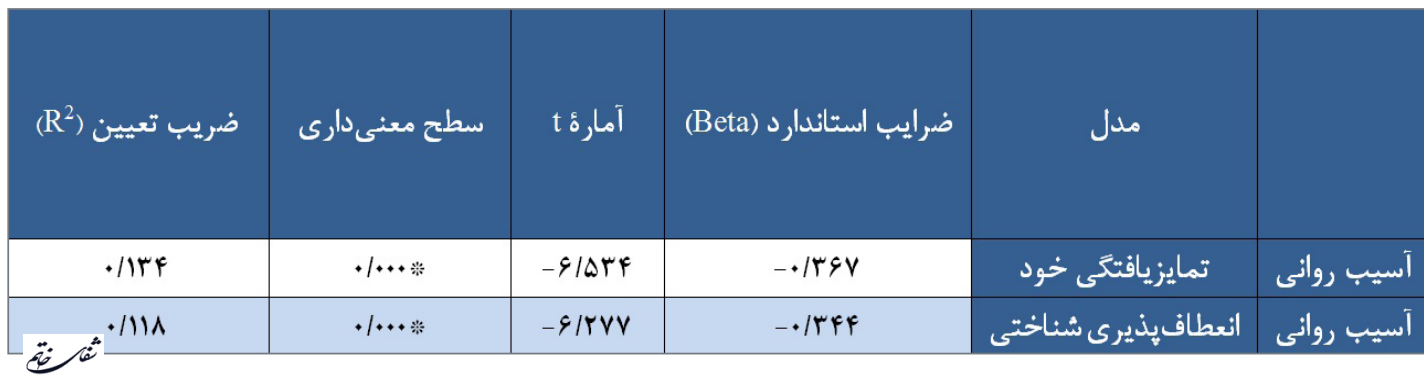

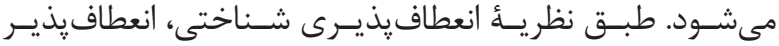

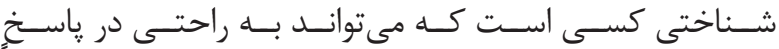

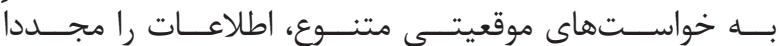

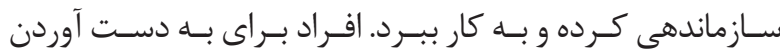

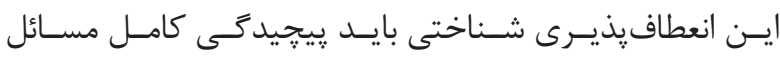

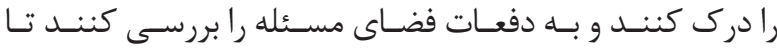

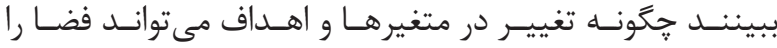

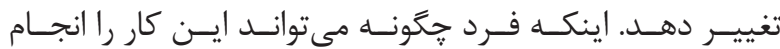

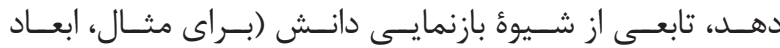

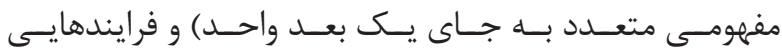

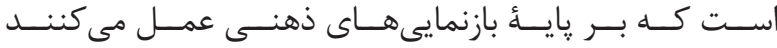

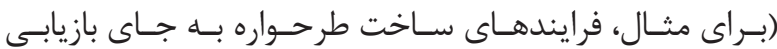

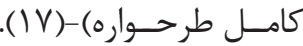

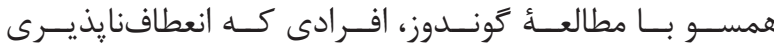

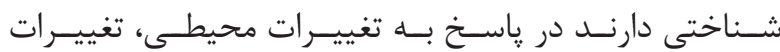

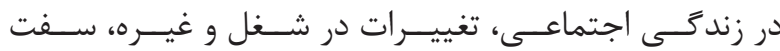

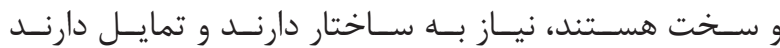

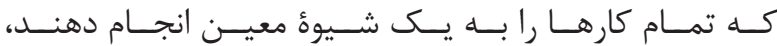

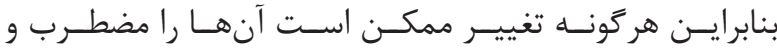

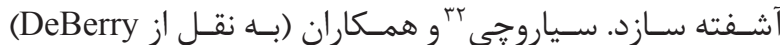

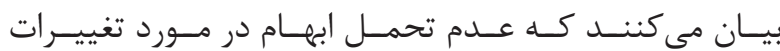

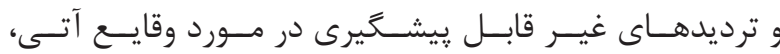

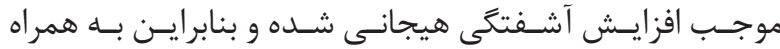

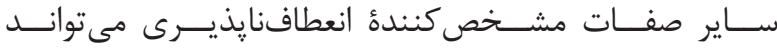

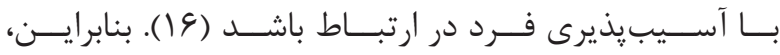

${ }^{29}$ Skowron, Stanley and Shapiro

${ }^{30}$ Sandage and Jankowski
بحث و نتيجه كيرى

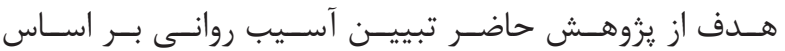

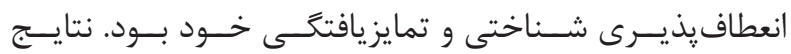

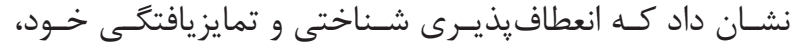

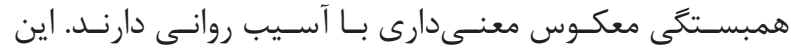

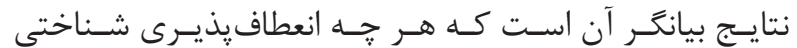

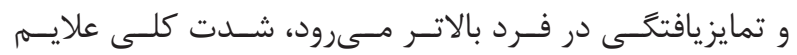

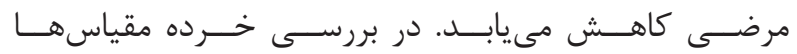

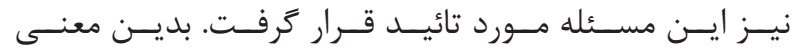

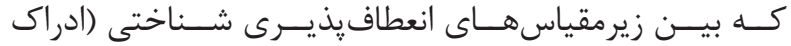

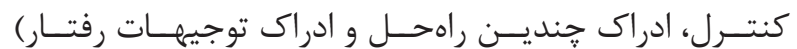

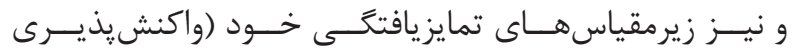

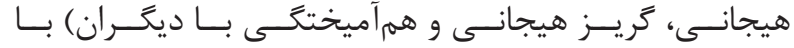

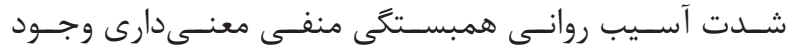

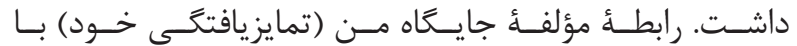

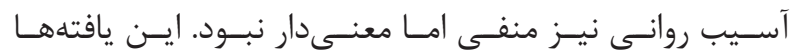

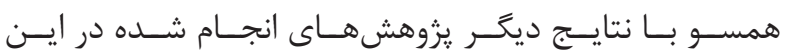

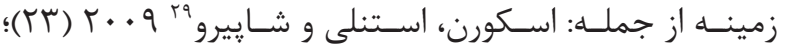

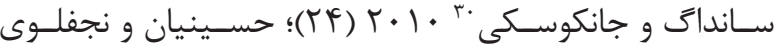

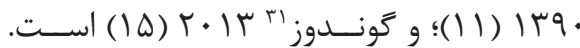

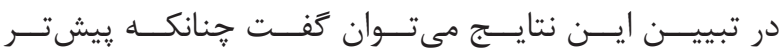

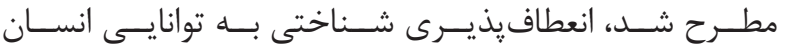

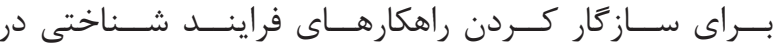

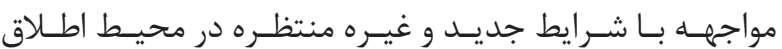

${ }^{31}$ Gunduz

${ }^{32}$ Ciarrocchi 


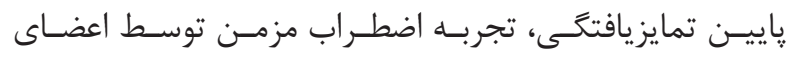

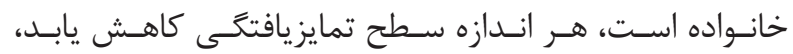

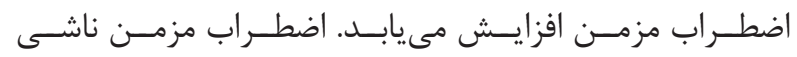

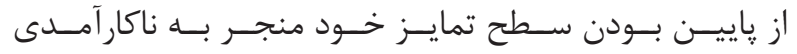

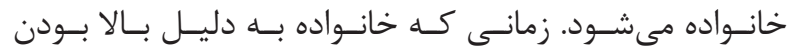

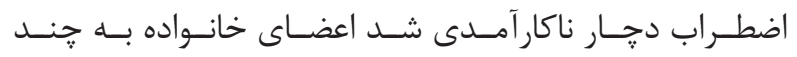

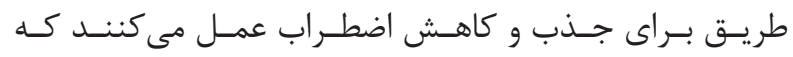

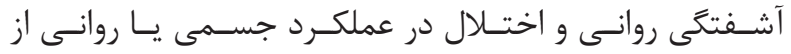

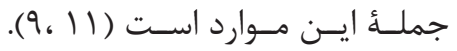
نتايــج ايسـن مطالعـه نشـان داد كـهـ بيـن آسـيب روانسى بــا

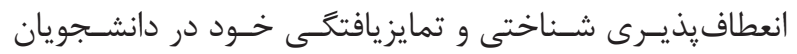

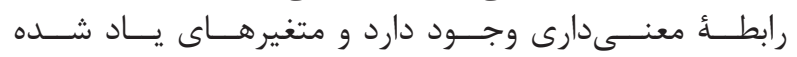

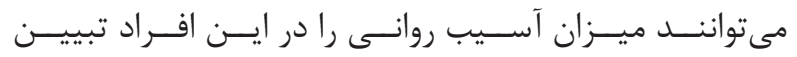

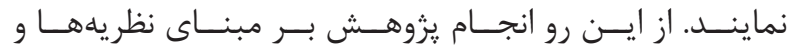

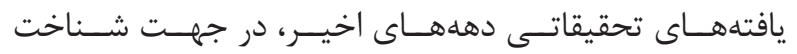

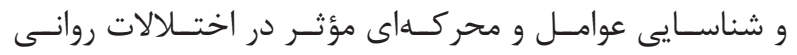

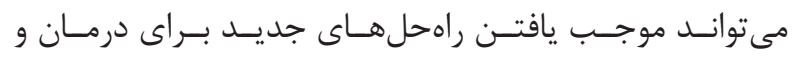

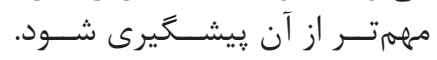

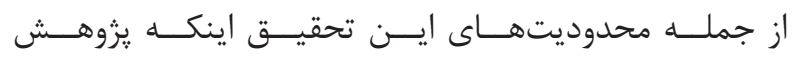

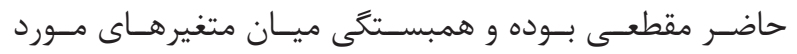

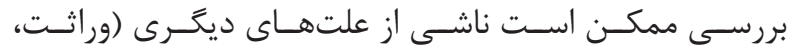

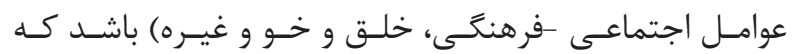

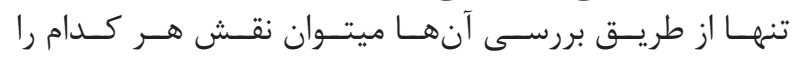

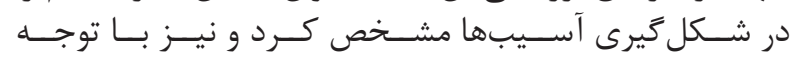

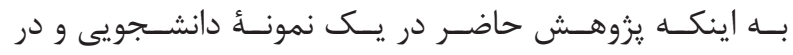

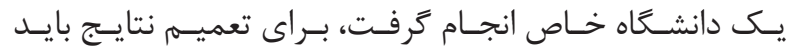

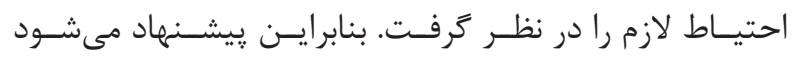

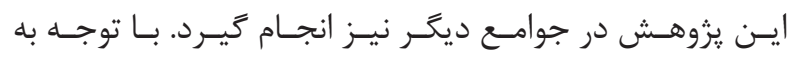

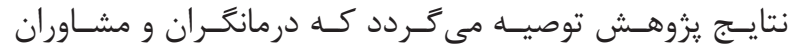

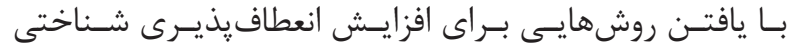

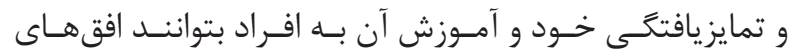

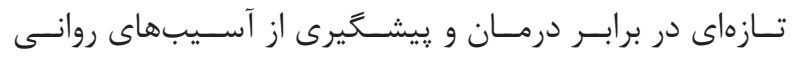
بخَشـايند.

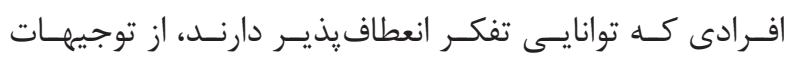

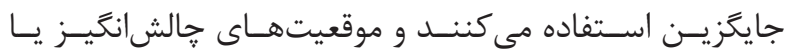

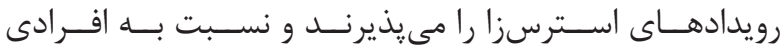

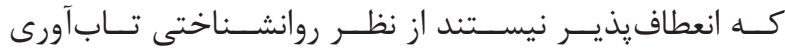

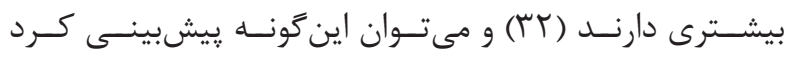

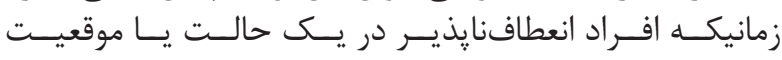

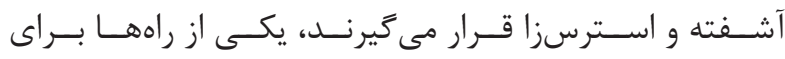

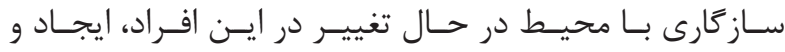

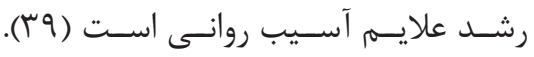

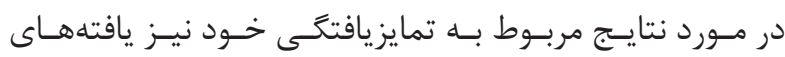

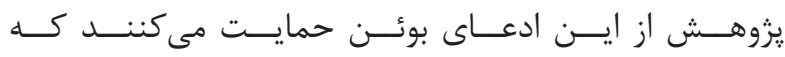

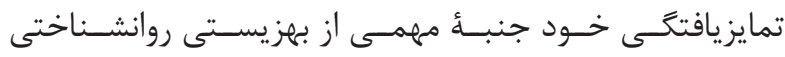

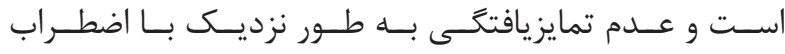

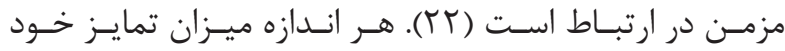

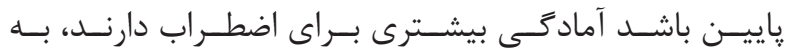

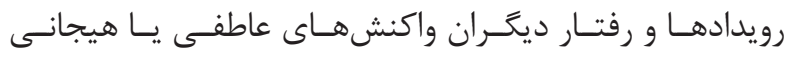

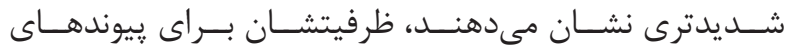

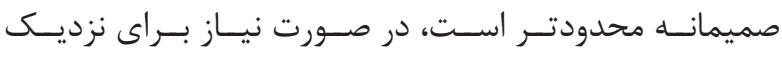

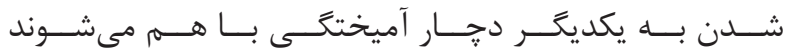

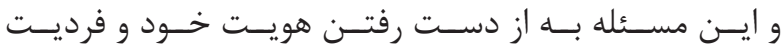

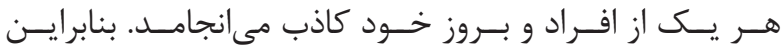

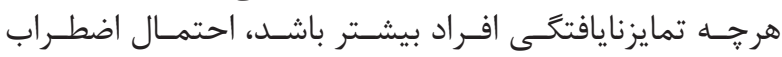

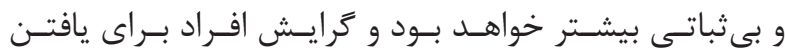

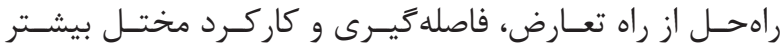

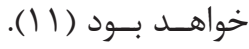

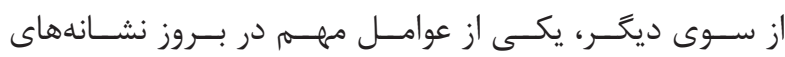

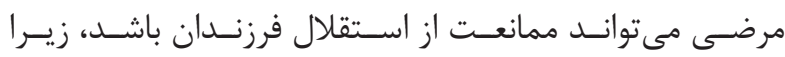

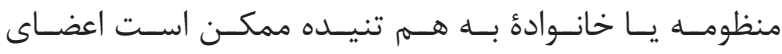

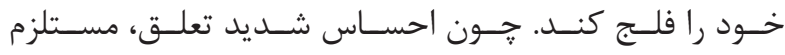

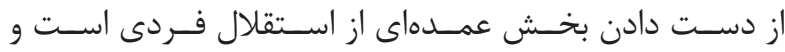

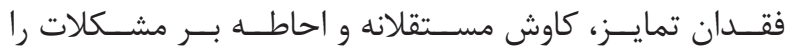

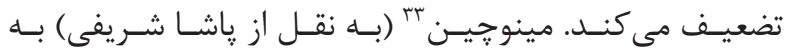

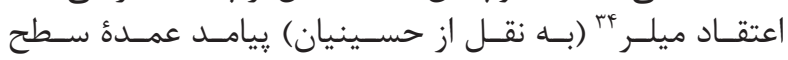


1. Garmezy N. Stressors of childhood. Garmezy N, M Rutter. Stress, coping, and development in children. New York: McGraw-Hill. 1983.

2. Harris AE, Curtin L. Parental perception, early maladaptive schemas and depressive symptoms in young adult. Cognit Ther Res. 2002; 26(3): 405-16.

3. Karami J, Zakiei A, Alikhani M, Nourouzi A. Explanation of self differentiation in accordance with attachment styles and alexithymia. J Behav Sci. 2014; 8(2): 139-47.

4. Bayrami M, Fahimi S, Akbari E, AmiriPichakolaei A. Predicting marital satisfaction on the basis of attachment styles and differentiation components. J Fundament Ment Health. 2012; 14(1): 64-77.

5. Peleg O, Zoabi M. Social anxiety and differentiation of self: a comparison of jewish and arab college students. Pers Individ Dif. 2014; 68: 221-8.

6. Tuason MT, Friedlander M. Do parent's differentiation levels predict those of their adult children? and other test of bowen theory in philippine sample. J Couns Psychol. 2000; 47(1): 27-35.

7. Skowron EA, Dendy Ak. Differentiae of self and attachment in adulthood: relational correlates of effortful control. J Contemp Fam Ther. 2004; 26(3): 337-57.

8. Skowron EA, Friedlander ML. The differentiation of self-inventory: development and initial validation. J Counsel Psychol .1998; 45(3): 235-49.

9. Pasha-Sharifi H, Manavipour D, Askari F. Psychometric properties of the differentiation of self inventory. J Ind/Organ Psychol. 2014; 5(18): 9-22.

10. Pirsaghi F, Nazari AM, Hajihassani M, NadAlipour $\mathrm{H}$. The effect of self differentiation therapy on assertiveness of female students of AllamehTabataba I University. J Clin Psychol Stud. 2014; 4(16): 19-34.

11. Hoseinian S, Najaflooy F. The relationship between differentiation of self with psychological and physical symptoms in women referring to counseling centers. J Women Fam Stud. 2011; 3(11): 29-44.

12. Goetter EM, Elizabeth M. An empirical investigation of depressive rumination: implications for cognitive flexibility, problem solving and depression. MA thesis. Philadelphia. Science Drexel University. 2010.
13. Dennis JP, Vander WalJ JS. The cognitive flexibility inventory: instrument development andestimates of reliability and validity. Cognit Ther Res. 2010; 34(3): 241-53.

14. Carbonella JY, Timpano KR. Examining the link between hoarding syptoms and cognitive flexibility deficits. Behav Ther. 2016; 47(2): 262-73.

15. Gunduz B. Emotional intelligence, cognitive flexibility and psychological symptoms in preserviceteachers. Educ Res Rev. 2013; 8(13): 1048-56.

16. DeBerry L. The relation between cognitive inflexibility and obsessive-compulsive personality traits in adults: depressionand anxiety as potential mediators. MA Theses. Southern Mississippi. University of Southern Mississippi. 2012.

17. Fazeli M, Ehteshamzadeh P, Hashemi Sheikh Bahani SE. The effectiveness of cognitive behavior therapy on cognitive flexibility of depressed people. J Thought Behav Clin Psychol. 2014; 9(34): 27-36.

18. Koesten J, Schrodt P, Ford DJ. Cognitive flexibility as a mediator of family communication environments and young adults' well-being. Health Commun. 2009; 24(1): 82-94.

19. Martin MM, Staggers SM, Anderson CM. (The relationshipsbetween cognitive flexibility with dogmatism, intellectual flexibility, preference for consistency, and self-compassion. Commun Res Rep. 2011; 28(3): 275-80.

20. Hooper LM, DePuy V. Mediating and moderating effects of differentiationof self on depression symptomatology in a rural community sample. Fam J. 2010; 18(4): 358-68.

21. Momeni KH, Alikhani M. The relationship between family functioning. differentiation of self and resiliency with stress anxiety and depression in the married women Kermanshah city. Fam Couns Psychother. 2013; 3(2): 297-320.

22. Peleg-Popko O. Bowen theory: a study of differentiation of self, social anxiety, and physiologicalsymptoms. Con temp FamTher. 2002; 24(2): 355-69.

23. Skowron EA, Stanley KL, Shapiro MD.Alongitudinal 
perspective ondifferentiation of self,interpersonal and psychological well-being inyoung adulthood. Contemp FamTher. 2009; 31(1): 3-18.

24. Sandage SJ, Jankowski PJ. Forgiveness, spiritual instability, mental healthsymptoms, and well-being: mediator effects of differentiation of self. Psychol Relig Spiritual. 2010; 2(3): 168-180.

25. Hosseinizadehm M. Examining the relationship between differentiation of self components and social adjustment. Procedia Soc Behav Sci. 2014; 113: 84-90.

26. Peleg O, Rahal A. Physiological symptoms and differentiation of self: a cross-cultural examination. Int J Intercult Relat. 2012; 36(5): 719-27.

27. Jenkins SM. Differentiation of self and psychology development. Contemp Fam Ther. 2005; 27(2): 251-61.

28. Brooks BL, Iverson GL, Sherman EM, Roberge MC. Identifying cognitive problems inchildren and adolescents with depression using computerized neuropsychological testing. Appl Neuropsychol. 2010; 17(1): 37-43.

29. Meiran N, Diamond GM, Toder D, Nemets B. Cognitive rigidity in unipolar depression and obsessive compulsive disorder: examination of task switching, strop, working memory updating and post-conflict adaptation. Psychiatry Res. 2011; 185(1-2): 149-56.

30. Preiss M, Kucerova H, Lukavsky J, Stepankova H, Sos P, Kawaciukova R. Cognitive deficits in the euthymic phase of unipolar depression. Psychiatry Res. 2009; 169(3): 235-9.

31. Watari K, Letamendi A, Elderkin-Thompson V, Haroon E, Miller J, Darwin C, et al. Cognitive function in adults with type 2 diabetes and major depression.
Arch Clin Neuropsychol. 2006; 21(8): 787-96.

32. Soltani E, Shareh H, Bahrainian SA, Farmani A. The mediating role of cognitive flexibility in correlation of coping styles and resilience with depression. Pejouhandeh. 2013; 18(2): 88-96.

33. Lee JK, Orsillo SM. Investigating cognitive flexibility as a potential mechanism of mindfulness in generalized anxiety disorder. J Behav Ther Exp Psychiatry. 2014; 45(1): 208-16.

34. Ornstein TJ, Arnold P, Manassis K, Mendlowitz $\mathrm{S}$, Schachar R. Neuropsychological performance in childhood OCD: a preliminary study. Depress Anxiety. $2010 ; 27(4)$ : 372-80.

35. Chamberlain SR, Fineberg NA, Blackwell AD, Robbins TW, Sahakian BJ. Motor inhibition and cognitive flexibility in obsessive- compulsive disorder and trichotillomania. Am J Psychiatry. 2006; 163(7): 1282-4.

36. Lysaker PH, Hammersley J. Association of delusions and lack of cognitive flexibility with social anxiety in schizophrenia spectrum disorders. Schizophr Res. 2006; 86(1-3): 147-53.

37. Shih JC, Farn CK, Ho CY. Conflict is not bad: interpersonal conflict and knowledge sharing. J Glob Bus Manage. 2008; 4(1): 46-57.

38. Deragotis LR. SCL-90-R: Administration, scoring and procedures manual-II for the revised version. 2nd ed. Towson. MD: Clinical Psychometric Research. 1983.

39. Fortin M, Hudon C, Bayliss E, Soubhi H, Lapointe L. Caring for body and soul:the importance of recognizing and managing psychological distress in persons with multi morbidity. Inter J Psychiatry Medic. 2007; 37(1): 1-9. 